\title{
O LIBERALISMO POLÍTICO DE RAWLS SOB UMA ÓTICA HEGELIANA: Contratualismo e o Aspecto Social nos Princípios de Justiça
}

\section{João Gilberto Engelmann}

Mestre em Filosofia. Pontifícia Universidade Católica do RS - PUCRS. razengel@gmail.com

\section{Resumo:}

0 texto foca duas questões principais que emergem da Conferência VII da obra Liberalismo político, de John Rawls: i) a defesa do contratualismo contra Hegel e ii) os princípios de justiça como refletores do aspecto social das relações humanas. 0 artigo usará, preponderantemente, as obras Elements of Philosophy of Right(Hegel) e Political Liberalism (Rawls). A tese central subjacente é a de que as aproximações que são possíveis entre Rawls e Hegel são tópicas e não sistemáticas, ou seja, Rawls e Hegel não comungam de uma mesma matriz teórica (identity of standpoint).

\section{Palavras-chave:}

Contratualismo. Hegel. Liberalismo. Rawls.

\section{RAWLS' POLITICAL LIBERALISM UNDER AN HEGELIAN VIEW:}

Social-Contract Theory and the Social Aspect of the Principles of Justice

\section{Abstract:}

the paper focuses on two main issues that emerge from the seventh lecture of John Rawls' Political Liberalism: i) the response to the Hegelian critique to social-contract theory and ii) the social aspect of human relations related to the principles of justice. This text dwells on Hegelian and Rawlsian works, mainly comparing both theories as presented in the Elements of Philosophy of Right (Hegel) and in the Political Liberalism (Rawls). The underlying 
central thesis which will base this analysis is that relations between Hegel and Rawls do not reveal more than topic and no systematic approaches as well as they do not share the same identity of standpoint.

\section{Keywords:}

Hegel. Liberalism. Social-contract theory. Rawls.

Recebido em: 4/6/2016

Aceito em: 11/8/2017

\section{Sumário:}

1 Introdução. 20 liberalismo político como uma teoria social-contratualista. 3 Princípios de justiça e as relações sociais dos indivíduos. 4 Considerações finais. 5 Referências. 


\section{INTRODUÇÃO}

O presente texto é um comentário sucinto acerca de alguns elementos da Conferência VII do Liberalismo político de John Rawls, ${ }^{1}$ parte esta em que se encontra, mais especificamente, um diálogo com Hegel. Tais são os elementos que pretendemos resgatar: i) a defesa do contratualismo contra Hegel e ii) os princípios de justiça como refletores do aspecto social das relaçóes humanas.

Em ambos os elementos está presente uma aproximação com Hegel, menos em termos de concessão à filosofia idealista e mais no sentido de afastar os prospectos do atomismo que circunscrevem a história da teoria contratualista como um todo (TAYLOR, 2001). Rawls, nesse sentido, não capitula à crítica idealista alocando o liberalismo político fora do campo contratualista. O que pretende, ao responder a Hegel, é justamente provar que o marco contratualista da justiça como equidade não implica recusa do elemento social das relaçóes humanas. Rawls é bem-sucedido nessa tarefa?

\section{LIBERALISMO POLÍTICO COMO UMA TEORIA SOCIAL-CONTRATUALISTA}

$\mathrm{Na}$ Conferência VII do Liberalismo político Rawls deseja aproximar a estrutura básica (primeiro objeto da justiça) às outras instituiçóes sociais e, nesse sentido, é natural que as objeçôes tradicionais de Hegel ao contratualismo sejam tratadas. ${ }^{2}$

Conforme apontada por Rawls ( $\$ 10)$, a crítica hegeliana ao contratualismo tem como ponto central o fato de a teoria do contrato social fazer confusão entre sociedade civil e Estado, concebendo este último sob a forma da associação de vontades privadas (RAWLS, 1996a, p. 322). Disso decorrem, ainda na visão

\footnotetext{
${ }^{1} \mathrm{Na}$ Conferência VII, os $\$ \$ 4-5$ sedimentam o teor primordial da estrutura básica da sociedade como primeiro objeto da justiça; $\$ \$ 6-7$ - o acordo inicial acerca dessa estrutura básica deve ser tomado como especial, distinto de quaisquer outros; o $\$ 8$ aproxima essa estrutura básica da sociedade (objeto primeiro da justiça e acordo especial) das relaçôes humanas sociais; $\$ 9$ os princípios que resultam objeto da posição original e que, portanto, são as figuras da estrutura básica da sociedade, devem materializar uma forma ideal dessa estrutura básica: limitação dos processos institucionais e ajuste das transaçóes pessoais; o $₫ 10$, ao reforçar os anteriores, reforça também a teoria contratualista presente no liberalismo político (na justiça como equidade).

2 Acerca da relação histórica e acadêmica entre liberais e comunitaristas, cf. Forst, 2010.
} 
hegeliana, outras duas consequências: forma e conteúdo do Estado estariam sendo determinados por interesses privados, ${ }^{3}$ que são contingentes à medida que variados; estaria eclipsado, além disso, o fato de que já nascemos em um Estado.

A paráfrase rawlsiana tem como base os seguintes textos de Hegel (1991, p. 275).

$\$ 258$ - If the state is confused with civil society and its determination is equated with the security and protection of property and personal freedom, the interest of individuals [der Einzehzen] as such becomes the ultimate end for which they are united; it also follows from this that membership of the state is an optional matter.

Outras consideraçôes precisas de Hegel em relação ao contratualismo, contudo, podem ser encontradas no $\$ 75$ e na continuidade do $\$ 258 .{ }^{4}$ No $\$ 75$ temse: "the nature of the state has just as little to do with the relationship of contract, whether it is assumed that the state is a contract of all with all, or a contract of all with the sovereign and the government" (HEGEL, 1991, p. 105).

$\mathrm{Na}$ segunda parte do $\$ 258$ :

The union of individuals [tier Einzebzen] within the state thus becomes a contract which is accordingly based on their arbitrary will and opinions, and on their express consent given at their own discretion; and the further consequences which follow from this, and which relate merely to the understanding, destroy the divine [element] which has being in and for itself and its absolute authority and majesty (HEGEL, 1991, p. 105). ${ }^{5}$

3 Acerca da distinção entre razão pública, social e privada, cf. nota 7 da Conferência VI (RAWLS, 1996a, p. 256).

${ }^{4}$ Consta, ainda, do $\$ 303$ dos Elements of Philosophy of Right (p. 344): “The many as single individuals - and this is a favourite interpretation of [ the term] 'the people' - do indeed live together, but only as a crowd, i.e. a formless mass whose movement and activity can consequendy only be elemental, irrational, barbarous, and terrifying. If we hear any further talk of 'the people' as an unorganized whole, we know in advance that we can expect only generalities and one-sided declamations".

5 Antes disso (p. 276), Hegel havia dito: "the philosophical approach deals only with the internal aspect of all this, with the concept as thought [mit dem gedaclzten Begriffi]. As far as the Ethical Lift search for this concept is concerned, it was the achievement of Rousseau to put forward the will as the principle of the state, a principle which has thought not only as its form (as with the social 
Tais são, genericamente, as objeçôes hegelianas à teoria do contrato social, ainda que haja nos Elements of Philosophy of Right uma concessão a Rousseau (nota de rodapé n. 8). ${ }^{6} \mathrm{O}$ fato que nos interessa, contudo, é o de o liberalismo político de Rawls ser justamente baseado numa concepção de contrato social e, portanto, se submeter à crítica hegeliana recém-transcrita (SMITH, 1986, p. 121).

Una teoría social-contractualista concibe el contracto original como fundador de un sistema de derecho público definidor y regulador de la autoridad política y vigente para todos y cada uno de los ciudadanos. Tanto la autoridad política como la ciudadanía han de entenderse a través de la concepción del contrato social mismo (RAWLS, 1996a, p. 301).

Rawls usa da crítica à teoria libertária para defender seu liberalismo político da pungente objeção hegeliana: "al entender el estado como una asociación privada, en cambio, la doctrina libertaria rechaza las ideas fundamentales de la teoría contratualista, y así [...] no deja margen a una teoría especial de la justicia para la estructura básica” (RAWLS, 1996a, p. 301). Está aí jacente a ideia de que o liberalismo político rawlsiano não concebe o Estado como uma associação privada. Como é possível, nesse sentido, conceber o liberalismo político como não atomista? Como são depurados os interesses particulares na posição original?

O aspecto atomista do contrato social é reduzido por sua própria caracterização: i) acordo entre todos ii) enquanto cidadãos, e não enquanto agentes desde um setor; iii) as partes se entendem como pessoas morais livres e iguais e iv) os conteúdos do acordo serão os primeiros princípios que regularão a estrutura básica (RAWLS, 1996a, p. 294). É evidente, para Rawls, que não se trata de atomismo conceber a tarefa de estruturar uma concepção política de justiça, sobretudo por

instinct, for example, or divine authority) but also as its content, and which is in fact thinking itself. But Rousseau considered the will only in the determinate form of the individual [einzelnen] will (as Fichte subsequendy also did) and regarded the universal will not as the will's rationality in and $\mathrm{f}$ or itself, but only as the common element arising out of this individual [einzebzen] will as a conscious will".

6 Acerca da crítica hegeliana ao uso do conceito de natureza humana dos contratualistas, cf. HEGEL, 1972. 
conta da reciprocidade do reconhecimento (pessoais morais livres e iguais) ${ }^{7}$ e pelo próprio objeto do contrato: um pano de fundo justo para o desenrolar dos processos sociais em curso na sociedade (RAWLS, 1996a, p. 303).

Em relação ao outro aspecto (interesses particulares na formatação do contrato) Rawls é bastante bem-sucedido na resposta a Hegel. O procedimento da posição original, conquanto se satisfaça em escolher os princípios de justiça que irão fundamentar a estrutura básica da sociedade, ${ }^{8}$ depura os interesses particulares mediante alguns instrumentos. Entre outros, o véu da ignorância e o consenso sobreposto. ${ }^{9}$

É perfeitamente compreensível que, dado o pluralismo de fato, não seja executável a tarefa de formatar uma estrutura básica de sociedade. ${ }^{10}$ As múltiplas justificaçôes pelas quais os indivíduos baseiam os respectivos comportamentos são concebidas dentro de morais abrangentes, ou seja, núcleos éticos distintos: cada qual com uma determinada e, às vezes, conflitante ideia de bem. Rawls afasta todo esse complexo de morais abrangentes por meio de uma delimitação: a delimitação do objetivo ou tarefa do liberalismo político (BROWNING, 1999, p. 132).

In a constitutional democracy one of its most important aims is presenting a political conception of justice that can not only provide a shared public basis for the justification of political and social institutions but also helps ensure stability from one generation to the next. Now a basis of justification that rests on self-or group-interests alone cannot be stable [and should be] dependent on a fortuitous conjunction of contingencies (RAWLS, 1999, p. 421, grifo nosso).

O Liberalismo político, por seu turno, assentará a tese de que não está suprimido o lastro ético que constitui a sociedade, o que é uma clara resposta ao elemento substancial reivindicado por Hegel (RAWLS, 1996a, p. 322). Não é

\footnotetext{
${ }^{7}$ A respeito da relação entre justiça e reciprocidade, cf. Rawls, 1999a, p. 190-224.

8 Acerca da independência da teoria moral da filosofia moral, cf. Rawls, 1999b, p. 286-302.

9 Dentre outros, cf. Rawls, 1999, p. 421; Rawls, 2001, p. 32; Rawls, 1996a, p 196.

${ }^{10}$ Acerca da ignorância quanto ao "estado atual da sociedade", cf. Rawls, 1996b, p. 308.
} 
possível, contudo, partir desse lastro ético para um consenso acerca dos elementos constitutivos (básicos) da sociedade. É perceptível, nesse sentido, o marcado teor metodológico (operacional) da teoria rawlsiana. ${ }^{11}$

Justice as fairness esclarece a condição social ou cultural que subjaz ao procedimento da posição original.

The three features [its requirements are limited to society's basic structure, its acceptance presupposes no particular comprehensive view, and its fundamental ideas are familiar drawn from the public political culture] allow different comprehensive views to endorse it. These include religious doctrines that affirm liberty of conscience and support the basic constitutional freedoms, as well as various liberal philosophical doctrines, such as those of Kant and Mill, that likewise do so (RAWLS, 2001, p. 33).

O que está bem sedimentado nessa passagem é que se exige somente um distanciamento das morais abrangentes em relação à posição original, e que a estrutura substancial que vige na sociedade permanece necessária, sobretudo porque o endosso aos princípios de justiça (que são o conteúdo da posição original) é o que suporta a teoria da justiça. É fundamental que o liberalismo político não confronte as morais abrangentes, como também não exija que os indivíduos adotem uma: "many citizens not hold any well-articulated comprehensive doctrine at all" (RAWLS, 2001, p. 33). Filosofias abrangentes como o construtivismo kantiano ou o utilitarismo podem perfeitamente endossar o liberalismo político, nesse sentido.

O que Rawls deseja esclarecer em relação à crítica idealista é, justamente, que do fato de o liberalismo político estar incluído em uma perspectiva contratualista não decorre a formação do pacto inicial como um arranjado de átomos ou como povoado por interesses privados. Mais uma vez Rawls é claro e bem-sucedido: pactos reais, ajustados a perspectivas contingentes, históricas e ligadas a interesses não públicos (individuais ou associativos) - como na proposta libertária (o exemplo é de Rawls) - não incapazes de fundar a instituição da estrutura básica da sociedade, notadamente marcada por seu background justo (RAWLS, 1996b, p. 301).

${ }^{11}$ Acerca do desenvolvimento da metodologia rawlsiana na filosofia política, cf. Silveira, Norman, 1995, p. 125-151. 
Seria possível, ainda, investigar a forma como uma visão hegeliana do político poderia recepcionar essa alternativa rawlsiana do contrato social. Poderíamos imaginar que a própria necessidade de se formatar uma estrutura básica para a sociedade, em si, já implica uma concepção errônea (em uma perspectiva hegeliana) do que constitui uma sociedade - a ideia de normatividade da estrutura básica e do próprio conceito de pessoa. Não é possível, contudo, desenvolver tal investigação aqui, pelo que nos limitamos a observar que a resposta de Rawls é, incialmente, bastante satisfatória.

\section{PRINCíPIOS DE JUSTIÇA E AS RELAÇÕES SOCIAIS DOS INDIVÍDUOS}

Para Rawls, o aspecto social das relaçôes humanas está refletido nos princípios de justiça (1996a, p. 314). O sucesso desse argumento é fundamental para neutralizar as críticas de uma visão hegeliana de justiça. Como é possível, no entanto, que os princípios de justiça, escolhidos por meio de um procedimento que exige determinado esvaziamento ético em relação à tarefa da posição original, possam refletir o aspecto social das relações humanas?

Vejamos como Rawls expóe a questão no $₫ 8$ da Conferência VII do Liberalismo político. "Consideremos ahora tres vías por las que el aspecto social de las relaciones humanas se refleja [is reflected] en el contenido de los principios mismos de justicia” (1996a, p. 314). Citemos na quase integralidade o argumento de Rawls, cujas três vias distinguidas devem explicitar a ideia de que o aspecto social das relaçóes humanas se reflete nos princípios de justiça.

Primeira via.

En primero lugar, el principio de diferencia (que regula las desigualdades sociales y económicas) no distingue entre lo que los ciudadanos adquieren en tanto que miembros de la sociedad y lo que podrían haber adquirido si no hubieron pertenecido a ella [...] Lo más que se sabe en la posición original es que todas las concepciones de la justicia que están disponibles para las partes tienen consecuencias mejores que el egoísmo general [...]. Ni nuestra situación el otras sociedades, ni el estado de naturaleza, pueden desempeńar el mejor papel en la 
estimación de las concepciones de la justicia. Y es claro que esas nociones son irrelevantes a la hora de aplicar los dos principios de justicia (RAWLS, 1996a, p. 314).

ii) Segunda via.

En segundo lugar, y relacionado con lo que se acaba de decir, los dos principios de justicia regulan el modo en que se adquieren los derechos a retribuciones a cambio de contribuciones a asociaciones, o a otras formas de cooperación, dentro de la estructura básica [....${ }^{12}$ No se pueden confundir esas contribuciones con contribuciones a la sociedad misma, o con el valor para la sociedad de sus miembros como ciudadanos [...] no hay lugar para la idea de una contribución individual a la sociedad análoga a la contribución individual a las asociaciones existentes en el seno de la sociedad. Si hay que comprar el valor de los ciudadanos, hay que decir que el valor de los mismos en una sociedad bien ordenada es siempre igual: y esa igualdad se refleja en el sistema de libertades básicas y de oportunidades equitativas, así como en la manera de operar del principio de la diferencia (RAWLS, 1996a, p. 315-316).

iii) Terceira via.

En tercer lugar, y por último, recuérdese que desde un punto de vista kantiano las partes son concebidas como personas morales libres y iguales. Decir que son personas morales es decir que poseen una concepción del bien (un sistema de objetivos finales) y una capacidad para entender una concepción de la justicia y para seguirla en el curso de su vida (un sentido de la justicia). Pues bien; la libertad de las personas morales puede interpretarse bajo dos guías: primero, en tanto que personas libres, se conciben a sí mismas en posesión de un interés de orden superior a la hora de regular el resto de sus intereses, incluidos sus intereses fundamentales, por medio de la razón, es decir, por medio de principios racionales y razonables que son expresión de su autonomía. Además, las personas

12 Parte suprimida: "como hemos visto, esas contribuciones se estiman sobre la base de los propósitos particulares de los individuos y de las asociaciones; y las contribuciones realizadas por la gente están en parte influidas por sus esfuerzos y logros, en parte por las circunstancias sociales y el azar. Las contribuciones sólo pueden definirse como contribuciones a esta o aquella asociación en esta o aquella situación. Tales contribuciones reflejan la utilidad marginal de un individuo a algún grupo particular" (RAWLS, 1996a, p. 314). 
libres no se conciben a sí mismas como indisolublemente vinculadas a ningún objetivo final particular, o a una familia de tales objetivos, sino que se entienden a sí propias como capaces en todo momento de juzgar y revisar sus propósitos a la luz de consideraciones razonables. En segundo lugar, partimos del supuesto de que las personas libres son responsables de sus intereses y de sus objetivos: son capaces de controlar y de revisar sus querencias y sus deseos, y, según lo pidan las circunstancias, aceptan la responsabilidad de hacerlo (RAWLS, 1996a, p. 316).

Comecemos por identificar semelhanças com Hegel em cada uma das vias transcritas: em i) está concordando com Hegel de que a hipótese de um estado de natureza não é útil à tarefa a que está adstrita a posição original. ${ }^{13}$ Contrariamente a Hegel, contudo, Rawls mantém o elemento contratual de que se deve levar a cabo um procedimento como o da posição original, o que, genericamente, não está presente em Hegel. Em ii) a igualdade emerge como no $\$ 36$ dos Elements of Philosophy of Right: todos são iguais de um modo formal (HEGEL, 1991, p. 69). Como em Hegel, há liberdades básicas $(\$ 66, \mathrm{PR}),{ }^{14}$ ainda que mais amplas, e oportunidades equitativas $(\$ 291, \mathrm{PR}),{ }^{15}$ ainda que não restritas aos cargos públicos.

Em iii) mantém-se relação com o $\$ 132$ da PR, sobretudo por sua explícita natureza kantiana: "the right of the subjective will is that whatever it is to recognize as valid should be perceived by it as good, and should be held responsible for an action as right or wrong, good or evil, legal or illegal, according to its cognizance of the value which that action has in this objectivity" (HEGEL, 1991, p. 158).

${ }^{13} \mathrm{O}$ argumento hegeliano é claro, entre outros, no artigo sobre o direito natural: "Suivant la fiction de l'état de nature, celui-ci est abandonné à cause des maux qu'il entraine, ce que ne signifie rien d'autre si ce n'est qu'est présupposé le terme où l'on veut arriver, à savoir qu'un accord [avec soi] serait le bien de ce qui est, en tant que chaos, en conflit [avec soi] serait le bien ou ce à quoi il faudrait parvenir" (HEGEL, 1972, p. 23, grifo nosso).

14 " $\$ 66$ - Those goods, or rather substantial determinations, which constitute my own distinct personality and the universal essence of my selfconsciousness are therefore inalienable, and my right to them is imprescriptible. They include my personality in general, my universal freedom of will, ethical life and religion” (HEGEL, 1991, p. 95).

15 “ $\$ 291-[\ldots]$ Individuals are not destined by birth or personal nature to hold a particular office, for there is no immediate and natural link between the two. The objective moment in their vocation [Bestimmullg] is knowledge and proof of ability; this proof guarantees that the needs of the state will be met, and, as the sole condition [of appointment], at the same time guarantees every citizen the possibility of joining the universal estate" (HEGEL, 1991, p. 322). 
Rawls está rechaçando, nas três vias, uma interpretaçáo atomizada dos princípios de justiça e da forma como eles se relacionam com o aspecto social das relaçóes humanas. Em i) isso é expresso pela ideia de que qualquer concepçáo de justiça que se apresente à posição original é melhor que o egoísmo geral (o egoísmo é, aqui, a marca do atomismo). Em ii) pela ideia de que a igualdade não é aferida e concedida, no contexto da estrutura básica, a partir do tanto que cada indivíduo contribuiu para o bem geral, mas sim a partir de um mínimo de condiçóes: liberdades básicas e oportunidade equitativas. No fundo significa: "la concepción política de la justicia [...] no puede consistir en el precepto: a las personas, de acuerdo con su virtud política” (RAWLS, 1996a, p. 112).

Por fim, em iii) Rawls pressupóe que as pessoas, concebidas como livres e iguais em um sentido kantiano não nutrem somente interesses pessoais imediatos, mas que, por conta da faculdade moral que lhes é exigida (ideia de bem e senso de justiça), tenham também interesses de "ordem superior". Tais interesses superiores, que influem justamente na consecução dos demais desejos individuais, podem ser descritos como preocupaçóes com uma ordem social estável na qual possam desenvolver seus objetivos - um sistema em que tais objetivos também se rejam por suas consequências e responsabilidades. Esse sistema superior de interesses faz com que as pessoas endossem os princípios de justiça como background justo - é um mínimo de ordem social para que a sociedade seja viável. Em sentido lato, esses interesses superiores náo teriam, segundo Rawls, um lastro atomista, mas uma preocupação social que as pessoas morais compartilham.

Nesse cenário, a liberdade sobre a qual Rawls está tratando concebe-se dentro do marco das instituiçôes sociais básicas: "ello es que la libertad, tal como se aplica a las instituciones sociales, significa una determinada configuración [pattern] de derechos y libertades" (RAWLS, 1996a, p. 316).Quer esclarecer que os princípios de justiça não se destinam somente a indivíduos isolados, mas à estruturação de uma justiça básica (background justice), o que faz com que o aspecto social das relaçóes humanas possa neles se refletir.

Mais do que isso, "la igual libertad significa que ciertas libertades y oportunidades básicas son iguales y que las desigualdades sociales y económicas son reguladas por principios convenientemente diseñados [adjusted] para mantener el valor equitativo de esas libertades" (RAWLS, 1996a, p. 316-317). O aspecto so- 
cial, imagina Rawls, está atendido à medida que a liberdade e a igualdade (os dois princípios de justiça) "exigen algún tipo de forma pública, y el contenido de los principios satisface esta expectativa" (1996a, p. 316-317). A forma pública, nesse sentido, faz com que se estenda a todos um mínimo de condiçóes sem quaisquer exigências prévias. É um valor moral igual (1996a, p. 316).

\section{CONSIDERAÇÕES FINAIS}

As aproximaçóes que anteriormente indicamos não são capazes se alocar Rawls e Hegel dentro de um mesmo marco teórico. O liberalismo político é uma concepção construtivista. É perceptível que uma visão hegeliana de justiça não poderia concordar menos com o procedimento da posição original

O problema de Rawls, dentro de uma réplica hegeliana, seria derivado do fato de imaginar que o conteúdo dos princípios da estrutura básica surja no ato de escolha, ou seja, que somente a partir daí adquire o caráter político. Sem uma desconsideração parcial dos elementos substanciais - que não é anulação nem mesmo a supressão desses elementos - não é possível formatar senão uma estrutura provisória, argumenta Rawls.

Para Hegel, princípios escolhidos para vigorar náo podem conduzir a sociedade a um fim específico em sentido normativo. A constituição positiva condensa, assim, normas (regras e princípios) que expressem o ethos (a substância ética) de uma sociedade específica, mas jamais programas que se definem por fins a serem perseguidos (a sociedade que se deseja ver construída). A constituição formal, nesse sentido, seria um conceito mais sociológico (descritivo) do que normativo (teleológico).

A ideia de encobrimento do estado presente da sociedade é, portanto, completamente contrária a Hegel.

\section{REFERÊNCIAS}

BROWNING, G. K. Hegel and the history of political philosophy. Nova York: MacMillan Press Ltd, 1999. 
FORST, R. Contextos da justiça. Trad. Denilson Luis Werle. São Paulo: Boitempo Editorial, 2010.

HEGEL, G. W. F. Des manières de traiter scientifiquement du droit naturel. Trad. B. Bourgeois. Paris: Librairie Philosophique, 1972.

. Elements of Philosophy of Right. Trad. H. B. Nisbet. Cambridge: Cambridge University Press, 1991.

RAWLS, J. El liberalismo politico. Trad. Antoni Domènech. Barcelona: Crítica, 1996a. . Political liberalism. New York: Columbia University Press, $1996 \mathrm{~b}$. . Collected papers. Edited by S. Freeman. Cambridge: Harvard University Press, 1999a. . A theory of justice. Revised edition. Cambridge: Harvard University Press, 1999b. - Justice as fairness: a restatement. Edited by Erin Kelly. Cambridge: Harvard University Press, 2001.

SILVEIRA, P.; NORMAN, W. Rawlsianismo metodológico: una introducción a la metodología dominante en la filosofía política anglosajona contemporánea. RIFP, p. 125-151, 1995. SMITH, S. B. Hegel's critique of liberalism. American political Science Review, v. 80, n. 1, p. 121-139, march, 1986.

TAYLOR, C. Atomism. In: AVINERI, S.; SHALIT, A. (Org.). Communitarianism and individualism. Oxford: Oxford University Press, 2001. 